\title{
Succinate links mitochondria to deadly bacteria in cystic fibrosis
}

\author{
Speranza Esposito ${ }^{1}$, Valeria Rachela Villella ${ }^{1}$, Federica Rossin ${ }^{2}$, Antonella Tosco ${ }^{3}$, Valeria Raia ${ }^{1,3}$, \\ Alessandro Luciani ${ }^{4}$ \\ ${ }^{1}$ European Institute for Research in Cystic Fibrosis, San Raffaele Scientific Institute, Milan, Italy; ${ }^{2}$ Department of Biology; University of Rome \\ "Tor Vergata”, Rome, Italy; ${ }^{3}$ Pediatric Unit, Department of Translational Medical Sciences, Regional Cystic Fibrosis Center, Federico II University \\ Naples, Naples, Italy; ${ }^{4}$ Institute of Physiology, University of Zurich, Zurich, Switzerland \\ Correspondence to: Alessandro Luciani. Institute of Physiology, University of Zurich, Winterthurerstrasse 190, CH-8057 Zurich, Switzerland. \\ Email: alessandro.luciani@uzh.ch. \\ Provenance: This is an invited article commissioned by the Section Editor Dr. Wan Wang (Medical Technology School, Xuzhou Medical University, \\ Xuzhou, China). \\ Comment on: Riquelme SA, Lozano C, Moustafa AM, et al. CFTR-PTEN-dependent mitochondrial metabolic dysfunction promotes Pseudomonas \\ aeruginosa airway infection. Sci Transl Med 2019. doi: 10.1126/scitranslmed.aav4634.
}

Submitted Nov 17, 2019. Accepted for publication Dec 02, 2019.

doi: $10.21037 / \mathrm{atm} .2019 .12 .49$

View this article at: http://dx.doi.org/10.21037/atm.2019.12.49

Cystic fibrosis (CF) is a life shortening-autosomal recessive disease caused by loss-of-function mutations in the gene encoding the CF transmembrane conductance regulator (CFTR) that transports essential electrolytes in and out of specialized cells (1). The loss of CFTR causes anomalies in the epithelial ion transport, leading to dehydration of cell surfaces and resulting in the accumulation of sticky obstructions that eventually destroy the integrity and physiology of different organ systems (2). The aberrant clearance of the thickened mucus, which normally traps one or more inhaled bacteria, triggers the chronic activation of non-resolving inflammation to cause lung damage that can eventually lead to respiratory failure in people with $\mathrm{CF}(3,4)$. Further clinically relevant manifestations associated with CF include dysfunction of gastrointestinal tract, hepatobiliary failure, and male infertility and increased chloride in sweat that represents a routinely biomarker for CF diagnosis (5-7).

To date, there is no cure for individuals with CF, and the only available treatments are directed to ease the detrimental consequences of CFTR dysfunction, restricting the therapeutic avenues to supportive care. Indeed, people with CF habitually take more than 40 medications a day including antibiotics, anti-inflammatory and inhaled mucolytic agents and spend at least $14 \mathrm{~h}$ a week doing physiotherapy and respiratory therapy to eradicate the early-stages of bacterial infections and relieve the respiratory manifestations (8). Pancreatic enzyme replacement and other supplements help prevent various nutritional deficiencies, hence decreasing the mortality and improving the survival of individuals born with CF (9). Despite the recent breakthrough came in the form of CFTR modulators (10), the disease still curbs the quality of the life for patients and for their families, highlighting the need of developing novel therapeutic options for efficiently attenuating this life-threatening disease.

Persistent hyper-inflammation and airway infections, mainly by Pseudomonas aeruginosa, Staphylococcus aureus, and Burkholderia cepacia, cause significant morbidity and mortality in patients with CF. The inability of the host airway cells to eradicate the different microbial species, which subsequently colonize within the lungs of patients, reflects the biofilm formation and the increasing occurrence of antimicrobial resistance (11). Besides functioning as a pure ion channel, recent pieces of evidence support the emerging vision that CFTR acts as a scaffold for several intracellular proteins and-by doing that-it helps chaperone properly them to their cellular compartments. For instance, through the binding to, and by repositioning the tumour suppressor phosphatase and tensin deleted on chromosome 10 (PTEN) (12) to the plasma membrane, CFTR seemingly contributes to the regulation of the immune PI3KAkt signalling cascade, thereby maintaining the host immunity against Pseudomonas aeruginosa infection (13). Strong evidence for this notion derives from the suppression 
of the anti-inflammatory PI3K activity in patients carrying CFTR mutations (14), and from the activation of hyperinflammatory responses in mice lacking Pten (13), which faithfully replicates the pathological inflammation seen in patients with CF. Therefore, the lack of CFTR subverts the capacity of the airway cells to tether efficiently PTEN to plasma membrane where it can participate in PI3Kmediated regulation of anti-bacterial responses, fuelling ultimately the CF inflammatory pathology. How the combined action of CFTR and PTEN anomalies synergizes to sustain the dysregulation of the airway inflammationand the specific contribution of this interaction to host immunity in the context of lung physiology and disease-is still a wide-open question.

The study by Riquelme and colleagues (15) sheds light on these questions by revealing a critical and mechanistically surprising role of CF mitochondria (e.g., the cellular power generators) and their derived metabolites in feeding deadly bacteria that evade the host immune system, leading to chronic infections that are almost impossible to eliminate and resulting ultimately lethal.

In their study, the authors selected a diverse panel of human epithelial and immune cell lines, mouse models of CF and Pten deficiency, and Pseudomonas aeruginosa isolates derived from patients with CF, and cogently analysed the homeostasis and functionality of mitochondrial network, either alone or after stimulation with pulmonary CF bacterial pathogens. Strikingly, Riquelme and colleagues revealed alterations in the function of the $\mathrm{CF}$ mitochondrial network and cellular metabolism that correlate with the downregulation of the gene encoding the PTEN protein. In fact, epithelial cells lacking PTEN displayed augmented mitochondrial and cellular assimilation of metabolites that predominantly trigger reactive oxygen species (ROS) production and oxidative stress through the complex I when compared to PTEN-competent cells. These changes in the mitochondrial redox homeostasis and function similarly recapitulated the mitochondrial abnormalities encountered in animal and cell-based CF models as well as in patientderived airway cells (16).

Several conditions, including bacterial infections and inflammation, stimulate the immunoresponsive gene 1 (IRG1) $(17,18)$ coding an enzyme that produces itaconitate through the decarboxylation of cis-aconitate, a tricarboxylic acid (TCA) cycle intermediate. In addition to the bactericide activity on opportunistic microbes, recent studies indicate that itaconitate metabolites drive posttranslational-related regulatory circuits that govern the remodelling of TCA cycle, hence the metabolic adaptation that accompanies the activation of the immune cells during inflammatory processes (19). Notably, the increased concentrations of itaconitate disable the enzyme activity of the mitochondrial succinate dehydrogenase $(\mathrm{SDH})$, whose inhibition elevates the amounts of succinate molecules, regulating ultimately the metabolic rewiring and the inflammatory cytokine production during macrophage activation (19). Based on these observations, Riquelme et al. determined whether the PTEN-driven mitochondrial ROS and metabolic alterations activate the IRG1-itaconate axis, forcing the defective mitochondria to release an excessive amount of succinate. To test this hypothesis, the authors combined both cell and animal-based models of Pten deficiency and stimulated the latter with a laboratory strain of Pseudomonas aeruginosa (PAO1) to induce the recurrent lung infections encountered in patients with CF. They found that the PTEN deficiency, either alone or in combination with the PAO1 microbes, leads to the activation of the antioxidant IRG1 pathway, both in vitro and in vivo, and that intracellular levels of itaconate heighten progressively, paralleling an increase in the concentrations of succinate metabolites from dysfunctional ROSproducing mitochondria. These cellular alterations did not result from abnormalities in the uptake of succinate within mitochondria, nor changes in the glutamine/GABA $(\gamma-$ aminobutyric acid) metabolism nor did anomalies in the hypoxia-induced factor $1 \alpha$ HIF $1 \alpha$ signalling, which were similar in both control and PTEN-depleted cells. These cumulative data suggest that the accumulation of succinate metabolites likely occurs through the PTEN-mediated dysregulation of ROS-IRG1-itaconate mitochondrial axis. Reinforcing the notion that PTEN plays a crucial role in maintaining mitochondrial network homeostasis, mouse knockout studies revealed that deletion of an isoform of Pten (Pten $\alpha$ ), which predominantly localizes in mitochondrial organelles, impairs PINK1/Parkin-mediated quality control of damaged and/or dysfunctional mitochondria, thereby triggering mitochondrial ROS production and oxidative stress, hence impairing cardiac homeostasis (20).

Having established that PTEN safeguards the mitochondrial functionality and cellular metabolism, the authors investigated whether the lack of PTEN function promotes the same chain of cellular events within the mitochondria of CF epithelial cells. To examine the role of PTEN in mitochondrial homeostasis and function, they engineered mutated $\left(C F T R^{\mathrm{F} 508 \mathrm{de} / \mathrm{F} 508 \mathrm{del}}\right) \mathrm{CF}$ cells to express either PTEN or the wild-type CFTR. Under the microscope, 
the functional expression of CFTR and PTEN at the outer mitochondrial membrane reinstates the homeostasis of the mitochondrial network, reducing ROS production and the activation antioxidant IRG1 pathway, hence bringing back the succinate-itaconate balance. Of note, similar excess of mitochondria-derived succinate metabolites was also observed in peripheral mononuclear blood cells and in airway fluid isolates derived from patients with $\mathrm{CF}$ as well as in a mouse model of CF ( $C f t r^{\text {F508del/F508del }}{ }^{\text {gut-corrected }}$ mice), either alone or after stimulation with PAO1 bacteria, and these metabolic changes correlated with impaired mitochondrial activity induced by the reduced expression of PTEN. Together, these findings provide further evidence for the authors' model supporting the concept that the CFTR dysfunction alters SDH activity, hence the levels of the succinate molecules through the dysregulation of the PTEN-IRG1 axis, which is crucial for preserving the redox homeostasis and immunoregulation.

The question remained how the augmented succinate levels could contribute to airway inflammation and chronic colonization of the CF lungs by Pseudomonas aeruginosa. Most bacteria can metabolize and utilize substrates from a mixture of different carbon sources, and the ability to select the preferred carbon sources is the driving force for safeguarding the evolution of free-living and pathogenic species (21). Among the many inadvertently inhaled pathogens, Pseudomonas aeruginosa bacteria are programmed to induce the accumulation of a large variety of metabolites and to preferentially catabolize succinate for sustaining their own growth and long-term colonization of host tissues (22). This bacterium's feeding preference raises one substantial question whether Pseudomonas aeruginosa would metabolize the succinate to favour bacterial growth and elicit long-term infections within the lungs of people with CF. Consistent with this idea, Riquelme and colleagues noticed that the exposure of PAO1 microbes to high concentrations of succinate (500 $\mathrm{mM}$ for 24 hours) leads to a substantial reprogramming of numerous genes encoding proteins that are involved in the finely tuned regulation of various aspects of succinate metabolism, redox homeostasis and production of polysaccharide-rich extracellular polymeric substances. This succinate-induced adaptation augments the extracellular production of a sticky slime that makes the pathogenic microbes more difficult to eradicate, favouring ultimately the establishment of intractable biofilm and bacterial infections while repelling the airway colonization by Staphylococcus aureus-another pathogenic bacterium that invades the lungs of individuals with CF. Supporting further the clinical relevance of this mitochondrial connection, the metabolic adaptation to succinate observed in vitro was also detected in longitudinal Pseudomonas aeruginosa isolates from a patient with CF. Intriguingly, these findings reported by Riquelme and colleagues are in line with recent studies revealing a substantial regulation of $P$. aeruginosa-driven mitochondrial dysfunction and damage in airway epithelial CF cells (23).

To further verify the detrimental properties of the metabolically—adapted Pseudomonas aeruginosa isolates, the authors stimulated either the Pten-deficient- or Cftr ${ }^{\mathrm{F} 508 \mathrm{del} /}$ F508del_gut corrected mice with the succinate-adapted Pseudomonas aeruginosa isolates from patients with CF. Strikingly, they unveiled that the patient-derived microbial isolates maintain the ability to trigger the overproduction of mitochondrial ROS, thereby evoking the recruitment of itaconate producing myeloid cells that lose their capacity to eradicate efficiently the microbial infection. The fact that succinate-fed bacteria suppress the host immune system, further hampering the body's ability to control infection, might in part explain the recurrence of persistent infections within the lungs of patients with CF. The authors therefore posit that the defective CFTR-PTEN axis alters the mitochondrial network and cellular metabolism, resulting in the augmented production of mitochondria-derived succinate molecules that accumulate in turn within the lungs (Figure 1). This environment creates a privileged niche that enhances airway inflammation and susceptibility for chronic infection by Pseudomonas aeruginosa microbes in patients with CF.

Though this study provides an exciting link between the physical and functional association of CFTR with PTEN, mitochondrial homeostasis, and energy metabolism, and regulation of the immune system and host defence, important questions remain and warrant further investigations. Culturing of epithelial or monocyte cell lines as well as those derived from patients, and the use of CF sputum-like media do not adequately recapitulate the in vivo complexity of inflamed airways with metabolically active host cells and proliferating bacteria, restricting the elucidation of the fundamental principles regulating the host-pathogen communication. The limitations of the traditional mouse models, which do not faithfully replicate the pathogenesis of bacterial infections encountered in patients with $\mathrm{CF}$, emphasize the need of novel animal models for better understanding the disease mechanisms at the organ-system level. The recent development of new genetic animal models (such as ferrets and pigs) (2), which 


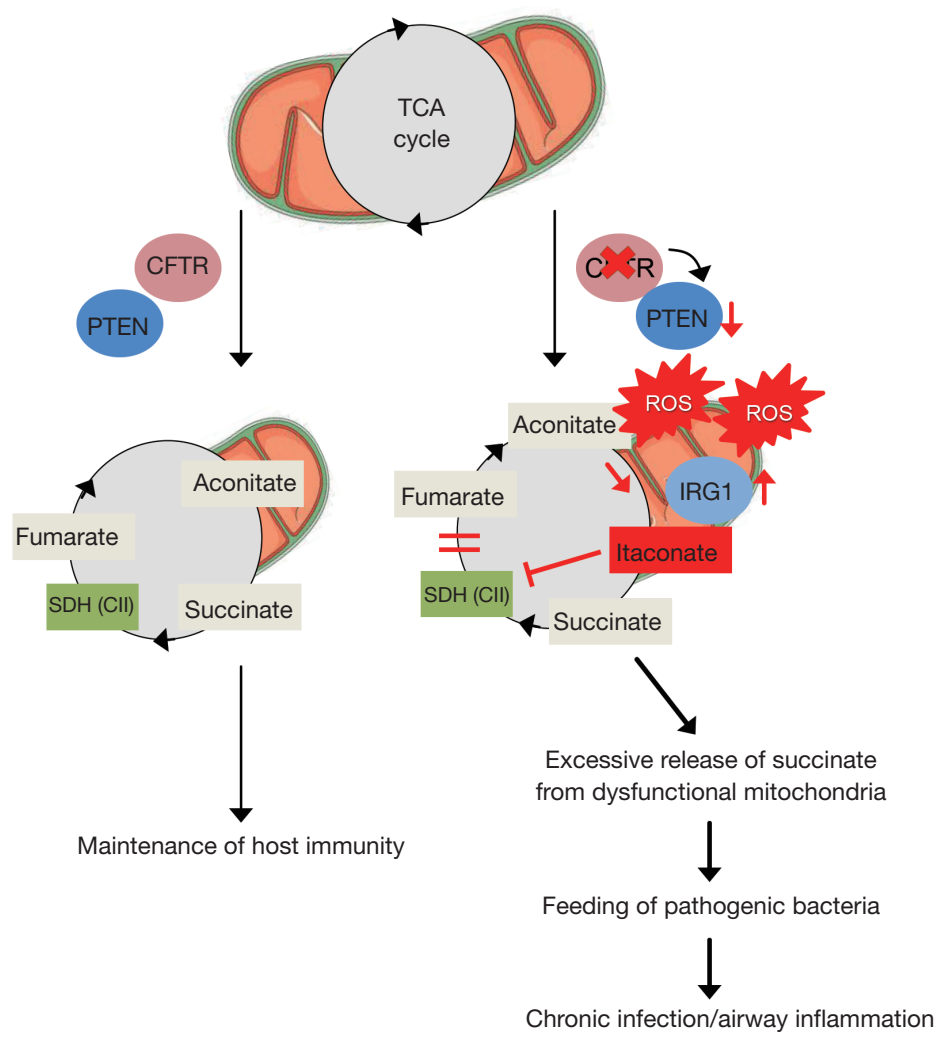

Figure 1 CFTR-PTEN dysfunctions drive mitochondrial metabolic defects that sustain Pseudomonas aeruginosa lung infection in CF. In wild type lung cells (left), the functional CFTR-PTEN axis preserves the mitochondrial energy metabolism, hence the homeostasis of the host immune system. In cystic fibrosis affected lung cells (right), the loss of CFTR-PTEN alliance triggers anomalies in the mitochondrial functionality and homeostasis, leading to ROS overproduction and activation of a compensatory antioxidant IRG1 response. This suppresses the mitochondrial SDH activity, resulting in the accumulation of succinate metabolites that feed the P. aeruginosa adaptation and microbial growth, hence promoting persistent infections and inflammation in the lungs of patients with CF.

reasonably approximate the lung physiology and develop severe lung disease at the earliest stages as in humans, might represent an opportunity to explore the in vivo role of CFTR-PTEN interactions in preserving the mitochondrial metabolism and the immune system homeostasis, and test eventually novel therapeutics.

Although the authors did not directly evaluate the potential impact of gut microbiome on lung immunity, these studies lay the groundwork for future research into whether, if any, how the microbiome-derived metabolites might sustain the homeostasis and function of the immune system, hence the dynamics of the host-microbe cross talk in the context of lung physiology and disease. It is tempting to speculate that CFTR-PTEN_driven maintenance of the mitochondrial energy metabolism might safeguard the milieu of the gut microbiome to set the tone of the immune system towards a healthy homeostasis. Accordingly, the ingestion of gluten proteins might switch the oral tolerance and subvert the host mucosal defences in a subset of genetically susceptible individuals bearing the human leukocyte antigen (HLA) DQ2/DQ, thereby causing inflammation and tissue damage in patients with celiac disease through the inhibition of the function of CFTR at the intestinal surface (24).

Beyond its role in transporting essential electrolytes in and out of specialized cells, CFTR serves also as a central hub that safeguards the cellular homeostasis by preserving the autophagy - mediated quality control of damaged and/ or dysfunctional organelles including mitochondria (16), hence controlling the adaptation to cell autonomous and/ or environmental stress signals. In this perspective, CFTR seems to sense the danger signals, alerting eventually the 
autophagy machinery when a stressful event threats the cellular homeostasis and physiology (16). In line with this concept, the long isoform of PTEN, which is known to be functionally impaired in $\mathrm{CF}$, seems to preserve the homeostasis and function of mitochondrial network in muscle cells through mitophagy-directed mitochondrial quality control systems (20). It would be interesting to investigate whether the FDA-approved CFTR modulators and/or alternative strategies targeting the repair of the mitochondrial dysfunctions and redox homeostasis (e.g., autophagy inducers and/or mitochondria-targeted ROS scavengers) $(20,25)$, restore the PTEN abundance, and the biological function of the complex, thereby preventing the feeding of succinate-adapted bacteria and their growth, hence dampening the exaggerated inflammation in the lungs of patients with CF.

All in all, the authors' discovery of succinate-fed bacteria provides a launch point for a deeper investigation of the roles of mitochondria-derived metabolites, which link the dynamic network of CFTR interacting components, mitochondrial function, and cellular metabolism to immune system homeostasis and host immunity. These clues potentially offer new opportunities for the development of next-generation therapeutics that could control the lung infections and airway inflammation, hence the respiratory health of people born with CF.

\section{Acknowledgments}

None.

\section{Footnote}

Conflicts of Interest: The authors have no conflicts of interest to declare.

Ethical Statement: The authors are accountable for all aspects of the work in ensuring that questions related to the accuracy or integrity of any part of the work are appropriately investigated and resolved.

\section{References}

1. Riordan JR. Assembly of functional CFTR chloride channels. Annu Rev Physiol 2005;67:701-18.

2. Cutting GR. Cystic fibrosis genetics: from molecular understanding to clinical application. Nature Rev Genet 2015; 16:45-56.
3. Stoltz DA, Meyerholz DK, Welsh MJ. Origins of cystic fibrosis lung disease. N Engl J Med 2015;372:351-62.

4. Ratjen F, Bell SC, Rowe SM, et al. Cystic fibrosis. Nat Rev Dis Primers 2015;1:15010.

5. Sanders DB, Fink AK. Background and epidemiology. Pediatr Clin North Am 2016;63:567-84.

6. Ramsey BW, Davies J, McElvaney NG, et al. A CFTR potentiator in patients with cystic fibrosis and the G551D mutation. N Engl J Med 2011;365:1663-72.

7. Cantin AM, Hanrahan JW. Thymosin $\alpha 1$ : a single drug with multiple targets in cystic fibrosis. Nature Med 2017;23:536-8.

8. Bell SC, Mall MA, Gutierrez H, et al. The future of cystic fibrosis care: a global perspective. Lancet Respir Med 2020;8:65-124.

9. MacKenzie T, Gifford AH, Sabadosa KA, et al. Longevity of patients with cystic fibrosis in 2000 to 2010 and beyond: survival analysis of the cystic fibrosis foundation patient registry. Ann Intern Med 2014;161:233-41.

10. Harutyunyan M, Huang Y, Mun KS, et al. Personalized medicine in CF: from modulator development to therapy for cystic fibrosis patients with rare CFTR mutations. Am J Physiol Lung Cell Mol Physiol 2018;314:L529-43.

11. Elborn JS. Cystic fibrosis. Lancet 2016;388:2519-31.

12. Bunney TD, Katan M. Phosphoinositide signalling in cancer: beyond PI3K and PTEN. Nat Rev Cancer 2010;10:342-52.

13. Riquelme SA, Hopkins BD, Wolfe AW, et al. Cystic Fibrosis Transmembrane Conductance regulator attaches tumor suppressor PTEN to the membrane and promotes anti Pseudomonas aeruginosa immunity. Immunity 2017;47:1169-81.e7.

14. Zhang PX, Cheng J, Zou S, et al. Pharmacological modulation of the AKT/microRNA-199a-5p/CAV1 pathway ameliorates cystic fibrosis lung hyperinflammation. Nat Commun 2015;6:6221.

15. Riquelme SA, Lozano C, Moustafa AM, et al. CFTRPTEN-dependent mitochondrial metabolic dysfunction promotes Pseudomonas aeruginosa airway infection. Sci Transl Med 2019. doi: 10.1126/scitranslmed.aav4634.

16. Luciani A, Villella VR, Esposito S, et al. Defective CFTR induces aggresome formation and lung inflammation in cystic fibrosis through ROS-mediated autophagy inhibition. Nature Cell Biol 2010;12:863-75.

17. Michelucci A, Cordes T, Ghelfi J, et al. Immune responsive gene 1 protein links metabolism to immunity by catalyzing itaconic acid production. PNAS 2013;110:7820-5.

18. Murphy MP, O’Neill LAJ. Krebs cycle reimagined: 
the emerging roles of succinate and itaconate as signal transducers. Cell 2018;174:780-4.

19. Lampropoulou V, Sergushichev A, Bambouskova M, et al. Itaconate links inhibition of succinate dehydrogenase with macrophage metabolic remodelling and regulation of inflammation. Cell Metab 2016;24:158-66.

20. Li G, Yang J, Yang C, et al. PTEN $\alpha$ regulates mitophagy and maintains mitochondrial quality control. Autophagy 2018;14:1742-60.

21. Görke B, Stülke J. Carbon catabolite repression in bacteria: many ways to make the most out of nutrients. Nat Rev Microbiol 2008;6:613-24.

22. O'Connell JD, Paulo JA, O'Brien JJ, et al. Proteome-wide

Cite this article as: Esposito S, Villella VR, Rossin F, Tosco A, Raia V, Luciani A. Succinate links mitochondria to deadly bacteria in cystic fibrosis. Ann Transl Med 2019;7(Suppl 8):S263. doi: 10.21037/atm.2019.12.49 evaluation of two common protein quantification methods. J Proteome Res 2018;17:1934-42.

23. Rimessi A, Bezzerri V, Patergnani S, et al. Mitochondrial Ca2+-dependent NLRP3 activation exacerbates the Pseudomonas aeruginosa-driven inflammatory response in cystic fibrosis. Nat Commun 2015;6:6201.

24. Villella VR, Venerando A, Cozza A, et al. A pathogenic role for cystic fibrosis transmembrane conductance regulator in celiac disease. EMBO J 2019;38:e100101.

25. Festa BP, Chen Z, Berquez M, et al. Impaired autophagy bridges lysosomal storage disease and epithelial dysfunction in the kidney. Nat Commun 2018;9:161. 